\title{
Electrospinning of radical polymers: redox-active fibrous membrane formation
}

\author{
Takeo Suga ${ }^{1,2}$, Matthew T Hunley ${ }^{3}$, Timothy E Long ${ }^{3}$ and Hiroyuki Nishide ${ }^{1}$ \\ Redox-active but highly durable nitroxide radicals, that is, 2,2,6,6-tetramethylpiperidinyl-4-oxy (TEMPO), enabled direct \\ electrospinning of radical-containing polymers without additional processing aids (such as polymer blends, post-doping, or \\ protection/deprotection) and produced redox-active fibrous membranes with high surface area. The solution rheological behavior \\ of the TEMPO-substituted polymethacrylate was similar to the neutral conventional polymers, and electrospinning of the radical \\ polymers yielded submicrometer-scaled fibrous membranes without any defects on the radical moiety. The obtained membrane \\ exhibited stable redox response, leading to redox catalysts or electrode-active materials toward organic-based flexible \\ rechargeable battery.
}

Polymer Journal (2012) 44, 264-268; doi:10.1038/pj.2011.120; published online 7 December 2011

Keywords: electrospinning; functional polymers; radical; redox

\section{INTRODUCTION}

Among stable organic radical molecules that possess an unpaired electron, nitroxide radicals (such as 2,2,6,6-tetramethylpiperidinyl-4oxy (TEMPO)) have attracted much attention due to their utilization as spin-labels, spin traps, and capping agents for controlled radical polymerization. ${ }^{1}$ Besides, based on the stable redox couple between the nitroxide radical and its oxidized form (oxoammonium cation, Scheme 1), nitroxide-functionalized polymers and organic/inorganic composites have been employed as heterogeneous redox reagents, which catalyze the oxidation of alcohols into aldehydes and ketones. ${ }^{2}$ This metal-free oxidation process has resurfaced as a green or environmentally-compatible chemical process. ${ }^{3}$

Recently, we have explored and utilized nitroxide polymers as cathode-active, charge-storage materials for secondary Li-ion batteries. $^{4-9}$ The organic radical battery composed of a radical polymer cathode has several advantages: high charge capacity (ca. $100-140 \mathrm{mAh} \mathrm{g}^{-1}$ ), high-charging and discharging rate performance (30 s for complete charge), and long cycle life often exceeding 1000 cycles. For both applications, to enhance the turnover cycles for catalysts or charge/discharge performance for electrode-active materials, construction of nitroxide supports with high surface area has an important role due to efficient mass (substrate or counter ion) transfer accompanied by the redox reaction. ${ }^{10}$ Prompted by the design of soft portable electronic equipments, such as roll-up displays and wearable devices, the development of bendable and flexible batteries has attracted increased interest. ${ }^{11}$ A fibrous membrane morphology improves mechanical flexibility and possesses an enhanced surface area to volume ratio.
Electrospinning is a simple and versatile technique to produce nano- and microfibrous membranes with high surface area and porosities from polymer fluids. These materials have attracted much interest for use as sensors, filters and tissue engineering scaffolds, and drug delivery membranes, among other applications. ${ }^{12-17}$ In the electrospinning process, a viscous, entangled polymer solution is ejected through a syringe toward a grounded target under the application of a high electric potential (typically $10-30 \mathrm{kV}$ ). The droplet of solution stretches into a thin jet during flight, and solvent evaporates rapidly from the jet, reducing its diameter and increasing the charge density on the surface. At a critical surface charge density, the jet undergoes a bending instability, resulting in chaotic whipping and stretching of the polymer jet before deposition on a grounded target (Figure 1).

Recently, electrospun fibrous membranes have been investigated in polyelectrolyte applications due to their high surface area for electrolyte absorption and fully interconnected pores, which can serve as efficient channels for ion conduction. ${ }^{18}$ The one-dimensionally aligned, nanostructured $\mathrm{LiCoO}_{2}$ and metal oxides prepared by solgel related electrospinning techniques have also exhibited superior electrochemical properties as electrode-active materials for smaller batteries. ${ }^{19,20}$ On the other hand, electrospinning of redox-active polymers, such as inherently conducting polymers in pure form, has been unsuccessful, ascribed to the intractability of doped state of the polymer (rigid structure and limited solubility). Instead, polymer blends with more readily electropun polymers, such as poly (ethylene oxide) or poly(vinylpyrrolidone), have been applied for electrospinning. ${ }^{21-24}$ In this study, we report, for the first time, the

${ }^{1}$ Department of Applied Chemistry, Waseda University, Tokyo, Japan; ${ }^{2}$ Research Institute for Science and Engineering, Waseda University, Tokyo, Japan and ${ }^{3}$ Department of Chemistry and Macromolecules and Interfaces Institute, Virginia Tech, Blacksburg, VA, USA

Correspondence: Professor H Nishide, Department of Applied Chemistry, Waseda University, Shinjuku, Tokyo 169-8555, Japan.

E-mail: nishide@waseda.jp

or Professor TE Long, Department of Chemistry and Macromolecules and Interfaces Institute, Virginia Tech, Blacksburg, VA 24061, USA.

E-mail: telong@vt.edu

Received 24 August 2011; revised 6 October 2011; accepted 10 October 2011; published online 7 December 2011 


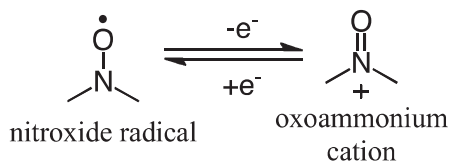

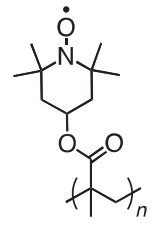

1

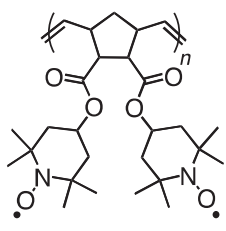

2
Scheme 1 Redox couple of a nitroxide and nitroxide polymers

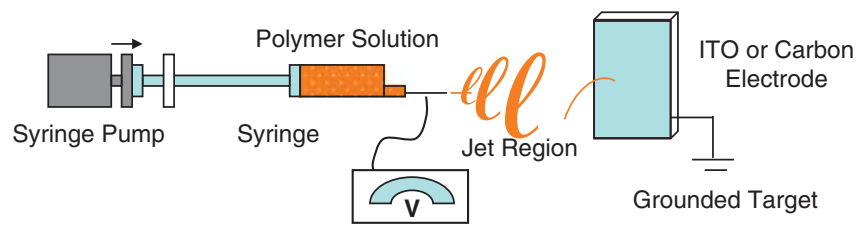

High Voltage Power Supply

Figure 1 Conventional solution electrospinning apparatus.

electrospinning of redox-active, nitroxide-containing polymers to prepare micrometer-scale fibrous membranes with high surface area. In addition to its application-oriented viewpoint, electrospinning of radical containing polymers is of interest from a fundamental point of view in terms of rheological studies of radical polymers and durability of radical sites under applied high electric potential, and contributes new insights into free radical chemistry. In combination with post-electrospinning photocrosslinking reactions, the insoluble radical-containing fibrous network was also prepared.

\section{EXPERIMENTAL PROCEDURE}

\section{Materials}

Poly(2,2,6,6-tetramethylpiperidin-4-yl methacrylate) $1 \quad\left(M_{\mathrm{w}}=74000\right.$, $M_{\mathrm{w}} / M_{\mathrm{n}}=1.4$ ) was obtained via anionic polymerization using phenylmagnesium bromide as an initiator. Synthetic details are followed by reference to our previous report. ${ }^{25}$ Poly(TEMPO-substituted norbornene) $2\left(M_{\mathrm{w}}=16000\right.$, $M_{\mathrm{w}} / M_{\mathrm{n}}=1.3$ ) was also synthesized via ring-opening metathesis polymerization by reference to our previous papers. ${ }^{26}$ Chloroform and $\mathrm{N}, \mathrm{N}$-dimethylformamide (DMF) were used as solvents without further purification.

\section{Electrospinning and fiber characterization}

Each polymer was dissolved in a $10 / 1 \mathrm{w} / \mathrm{w}$ mixture of $\mathrm{CHCl}_{3} / \mathrm{DMF}$ at the desired concentrations (7.5-20 wt $\%$ ) and mixed for $24 \mathrm{~h}$ before electrospinning. The electrospinning apparatus consisted of a high-voltage power supply (Spellman, Hauppauge, NY, USA, CZE-1000R), a syringe pump (KD Scientific, Holliston, MA, USA), a grounded target (stainless steel mesh) and an acrylic housing to reduce the effect of outside air currents. Electrospinning was conducted at ambient conditions. The solution was transferred to a syringe equipped with a stainless steel, 18-gauge needle. The syringe was placed in the syringe pump and solution metered at $3 \mathrm{ml} \mathrm{h}^{-1}$. The positive lead of the highvoltage power supply was connected to the syringe needle and potential increased to $25 \mathrm{kV}$. Fibers collected on the grounded target $20 \mathrm{~cm}$ away. For microscopy, a LEO 1550 field emission scanning electron microscope was used with an accelerating voltage of $5 \mathrm{kV}$. At least 20 individual fibers were measured using the SEM software to determine average fiber diameters and s.d.

\section{Polymer characterization}

Size-exclusion chromatography was performed at $40^{\circ} \mathrm{C}$ in high-performance liquid chromatography grade tetrahydrofuran at $1 \mathrm{ml} \mathrm{min}^{-1}$ using a Waters size-exclusion chromatographer equipped with an autosampler, three in-line 5-mm PLgel MIXED-C columns (Polymer Laboratories Inc., Amherst, MA, USA). Detectors included a Waters 410 differential refractive index detector operating at $880 \mathrm{~nm}$, and a Wyatt Technologies (Santa Barbara, CA, USA) miniDAWN multiangle $690-\mathrm{nm}$ laser light scattering detector. All reported molecular weight values are absolute molecular weights obtained using the multiangle $690-\mathrm{nm}$ laser light scattering detector. Solution rheology was performed on a TA Instruments (New Castle, DE, USA) AR-G2 rheometer, using concentric cylinder geometry. Temperature was maintained at $25 \pm 0.1{ }^{\circ} \mathrm{C}$ throughout the experiment. Steady shear measurements were performed from 1 to $100 \mathrm{~s}^{-1}$. Zero-shear viscosity was taken to determine specific viscosity at each concentration.

\section{ESR and SQUID measurement}

Electron spin resonance (ESR) spectra were taken using a JEOL JES-TE200 ESR spectrometer (JEOL, Tokyo, Japan) with a $100 \mathrm{kHz}$ field modulation. Magnetization and magnetic susceptibility of the powder polymer sample were measured by a Quantum Design MPMS-7 SQUID (superconducting quantum interference device) magnetometer. The magnetic susceptibility was measured from 10 to $300 \mathrm{~K}$ in a $1.0 \mathrm{~T}$ field. The radical density or the concentration of the unpaired electron of each sample was determined on the basis of the assumption of being paramagnetic at room temperature by integration of the ESR signal standardized with that of the TEMPO solution. The radical density was also analyzed by the slope of Curie plots and saturated magnetization in the SQUID measurement.

\section{Carbon electrode preparation}

A graphite fiber (vapor grown carbon fiber: vapor grown carbon fiber, Showa Denko Co., Tokyo, Japan, $80 \mathrm{mg}$ ) and $10 \mathrm{mg}$ of a binder powder (polyvinylidenefluoride resin: KF polymer, Kureha Chemical Co., Tokyo, Japan) were mixed in the presence of $\mathrm{N}$-methyl-2-pyrrolidone. The resulting black clay was spread onto an aluminum plate and dried in vacuo at $80^{\circ} \mathrm{C}$.

\section{Preparation of electrospun fibrous electrode 2}

To a chloroform/DMF $(10 / 1 \mathrm{w} / \mathrm{w})$ solution of polymer $2(15 \mathrm{wt} \%), 2,6$-bis ( $p$-azidobenzal)-4-t-amylcyclohexanone (BAC-TA, Toyo Gosei Co., Ltd, Tokyo, Japan, $1.5 \mathrm{wt} \%$ ) was added and the mixed solution was electrospun on a carbon or an indium tin oxide (ITO) electrode. After UV irradiation (Ushio Inc., Tokyo, Japan, USH-250D, $40 \mathrm{~mJ} \mathrm{~cm}^{-2}$ ), the electrode was washed with chloroform to remove any soluble species.

\section{Electrochemical measurements}

Cyclic voltammetry was performed using a normal potentiostat system (BAS Japan, Inc., Tokyo, Japan, ALS660B). A beaker cell with a three electrode system was prepared under an argon atmosphere and used for electrochemical measurements.

\section{RESULTS AND DISCUSSION}

Solution-processable poly(TEMPO methacrylate) 1 was synthesized via anionic polymerization of the corresponding radical monomer by reference to our previous reports. ${ }^{25}$ Before electrospinning process, we investigated solution rheology of polymer $\mathbf{1}$. Polymer $\mathbf{1}$ was soluble in chloroform, tetrahydrofuran and DMF, and studied using solution rheology to investigate its rheological behavior and to identify the critical concentration for entanglement, $C_{\mathrm{e}}$. Shear rate sweeps from 20 to $100 \mathrm{~s}^{-1}$ were performed on polymer solutions ranging from 7 to $20 \mathrm{wt} \%$ in a $10 / 1 \mathrm{w} / \mathrm{w} \mathrm{CHCl}_{3} / \mathrm{DMF}$ cosolvent at $25^{\circ} \mathrm{C}$. All polymer solutions showed Newtonian behavior over this shear rate range; however, it should be noted that shear thinning was expected to occur at higher shear rates in solutions above $C_{\mathrm{e}}$. The zero shear rate viscosity $\left(\eta_{0}\right)$ increased from 3.3 to 49.5 ( $\mathrm{mPa} \mathrm{s}$ ) as the concentration increased from 7 to $20 \mathrm{wt} \%$. The polymer contribution to the $\eta_{0}$ was studied by defining the specific viscosity $\left(\eta_{\mathrm{sp}}\right)$ in the following equation: $\eta_{s p}=\left(\eta_{0}-\eta_{s}\right) / \eta_{s}$, where $\eta_{s}$ is the solvent viscosity. For neutral, linear polymers in a good solvent, specific viscosity scales with concentration as $\eta_{\mathrm{sp}} \sim C^{1.0}$ in the dilute regime, $\eta_{\mathrm{sp}} \sim C^{1.25}$ in the semidilute unentangled regime, $\eta_{\mathrm{sp}} \sim C^{4.8}$ in the semidilute entangled regime, and $\eta_{\text {sp }} \sim C^{3.6}$ in the concentrated regime. Specific viscosity showed two distinct regimes with scaling exponents of 1.54 and 4.37 . These exponents are in good agreement with those expected for 
semidilute unentangled and semidilute entangled regimes, respectively, which suggested the stable free radicals do not contribute significantly to intermolecular interactions or flow behavior, and the radical polymer solution behaved as a non-associating, neutral polymer solution (Figure 2). The $C_{\mathrm{e}}$ was determined to be $12.4 \mathrm{wt} \%$, based on the change in slope using the method employed by Colby et al. ${ }^{27,28}$

Poly(TEMPO methacrylate) 1 was electrospun from a $10 / 1 \mathrm{w} / \mathrm{w}$ $\mathrm{CHCl}_{3} / \mathrm{DMF}$ cosolvent. Polymer 1 was soluble in $\mathrm{CHCl}_{3}$ at high concentrations; however, the rapid evaporation of chloroform during electrospinning resulted in plugging of the syringe injection nozzle. Therefore, DMF was used as a cosolvent to control the desired solubility and volatility. The fiber morphology was also varied with the processing conditions (applied potential, feed rate of the solution, and distance between the spinneret and the grounded target); however, earlier studies of one of our co-author (TE Long) have revealed the correlation between solution viscosity and fiber diameters of conventional polymers and polyelectrolytes. On the basis of the critical

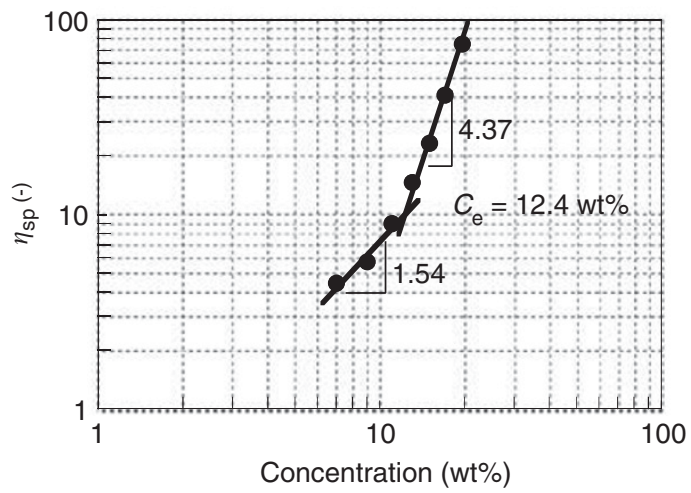

Figure 2 Dependence of specific viscosity on concentration for poly(TEMPO methacrylate) $1\left(M_{w}=74000\right)$. A full color version of this figure is available at Polymer Journal online. effects of solution viscosity on fiber morphology, other processing conditions ( $25 \mathrm{kV}$ potential, $3 \mathrm{ml} \mathrm{h}^{-1}$ flow rate from the syringe and a 20 - $\mathrm{cm}$ distance from the syringe tip to the grounded target) were held constant to discern the effects of solution concentration on morphology of the electrospun fibers.

Figure 3 shows field emission scanning electron microscope images of fibers electrospun using $7.5-18 \mathrm{wt} \%$ solutions of 1 . The fiber morphology was dependent on the concentration regime from which the polymer was electrospun. The polymer solutions electrospun from solutions below $C_{\mathrm{e}}(7.5 \mathrm{wt} \%)$ did not yield fibers, and only polymer droplets were observed, due to the lack of topological constraint or entanglements between polymer chains in solution in this concentration regime (Figure 3a). For the $10 \mathrm{wt} \%$ solutions near $C_{\mathrm{e}}$, beaded electrospun fibers were generated with diameters on the order of $0.3 \mu \mathrm{m}$ (Figure $3 \mathrm{~b}$ ). Above $C_{\mathrm{e}}$, the high degree of chain overlap resulted in chain constraint and the formation of numerous entanglements. For the 15 and $18 \mathrm{wt} \%$ solutions, uniform fibers (without any droplets or beaded fibers) with an average diameter of 3 and $5 \mu \mathrm{m}$ were formed (Figures $3 \mathrm{c}$ and $\mathrm{d}$ ). The fiber diameters depend on the polymer solution properties (chemical structure, solvent, $T_{\mathrm{g}}$, and concentration) and processing variables (applied electric potential). In general, as the number of entanglements increased, the solution viscosity increased, thereby facilitating the formation of uniform fibers with larger diameters. The interlaying of fibers generated a microporous structure with a high surface area.

It should be noted that the radical density of polymer 1 ( 0.94 radical per monomer unit) remained unchanged after electrospinning $(0.95$ radicals), which was estimated from the intensity of the ESR signal and from SQUID measurements, respectively. Surprisingly, the TEMPO group was sufficiently durable to be electrospun under high-applied potential $(25 \mathrm{kV})$, which was attributed to the undoped, neutral polymer, characteristic of the radical polymer in contrast to the conducting polymer.

When using radical polymers as electrode-active materials, it is crucial to eliminate dissolution of the radical polymer into the
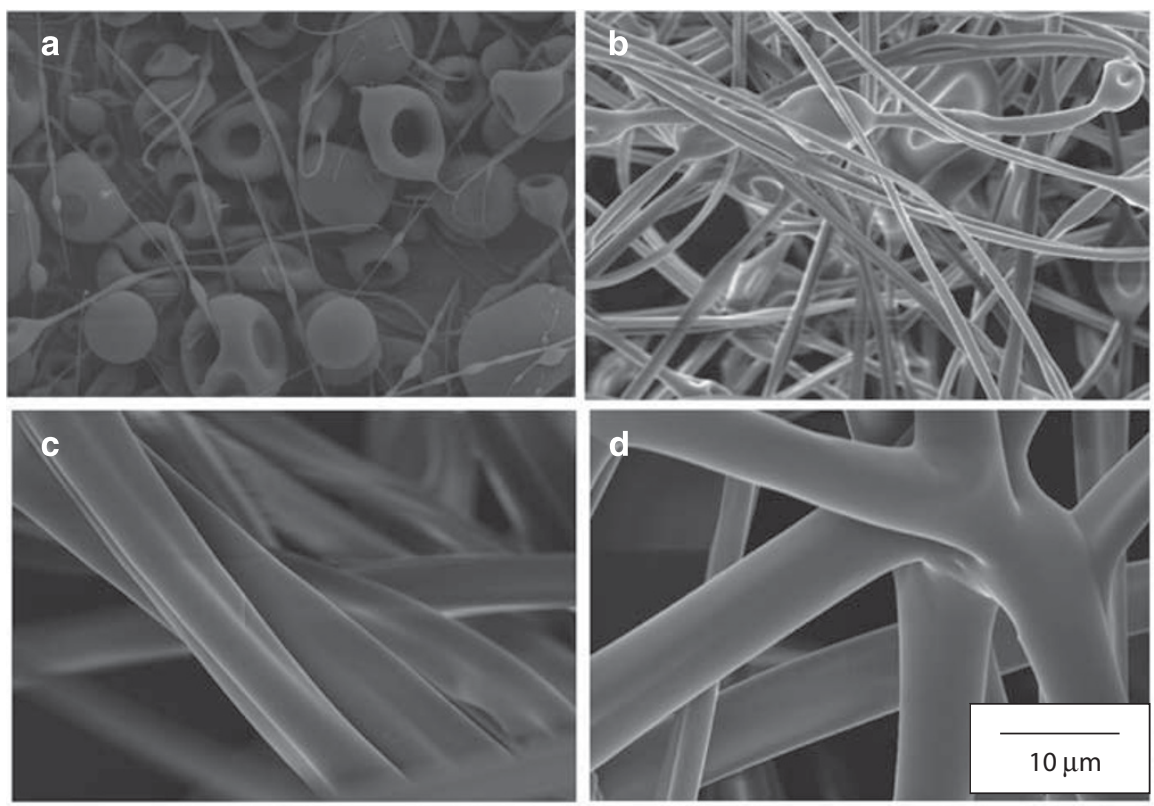

Figure 3 FESEM images of electrospun fibers of 1 from 10/1 w/w $\mathrm{CHCl}_{3} / \mathrm{DMF}$ solutions at several concentrations, (a) $7.5 \mathrm{wt} \%$; (b) $10 \mathrm{wt} \%$; (c) $15 \mathrm{wt} \%$; (d) $18 \mathrm{wt} \%$. 
a

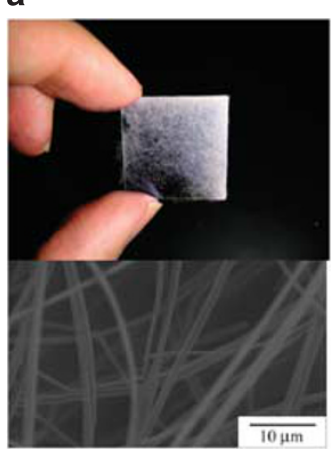

b

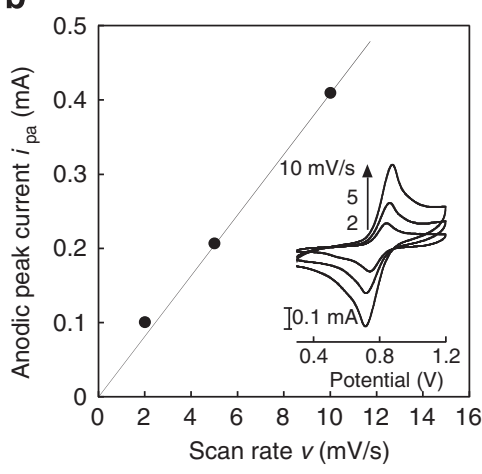

Figure 4 (a) Optical and field emission scanning electron microscope images of electrospun fibers of 2 from $10 / 1 \mathrm{w} / \mathrm{w} \mathrm{CHCl}_{3} / \mathrm{DMF}$ solutions (15 wt\%). (b) Cyclic voltammogram of photocrosslinked polymer fiber 2 on vapor grown carbon fiber electrode in $0.5 \mathrm{M}\left(\mathrm{C}_{4} \mathrm{H}_{9}\right)_{4} \mathrm{NClO}_{4}$; the counter electrode: Pt wire; the reference electrode: $\mathrm{Ag} / \mathrm{Ag}^{+}$; scan rate $=2,5$ and $10 \mathrm{mVs}^{-1}$.

electrolyte solution, which results in self-discharging of the battery. An insoluble fibrous membrane structure is also advantageous, as catalyst can be re-used or recycled. Therefore, electrospinning was applied for the photocrosslinkable poly(TEMPO-substituted norbornene) 2, which was prepared via ring-opening metathesis polymerization $\left(M_{\mathrm{w}}=16000, M_{\mathrm{w}} / M_{\mathrm{n}}=1.3\right){ }^{4}$ Polymer 2 was electrospun from a $15 \mathrm{wt} \%$ solution $\left(10 / 1 \mathrm{w} / \mathrm{w} \mathrm{CHCl}_{3} / \mathrm{DMF}\right.$ cosolvent) to yield a uniform fibrous membrane with an average diameter of $0.9 \mu \mathrm{m}$. A small amount of the bis(azide) derivative $(1.5 \mathrm{wt} \%)$ was added into a solution of polymer 2 as a photo-crosslinker. After electrospinning, UV irradiation with a high-pressure mercury lamp $\left(80 \mathrm{~mJ} \mathrm{~cm}^{-2}\right)$ induced nitrene formation, which resulted in reaction with the olefinic moieties of the polynorbornene backbone to afford a crosslinked fiber (Figure 4a). The obtained fiber $\mathbf{2}$ was insoluble, but slightly swollen in organic solvents. The crosslinked fiber became slightly yellowish due to the azo-crosslinker. The SQUID measurement of the collected fiber sample revealed 1.95 radicals per monomer unit, which suggested that side reactions on the TEMPO radical moiety did not occur during the photocrosslinking.

Electrochemical studies were performed with the electrospun radical fiber 2, which were collected on an ITO or vapor grown carbon fiber electrode as the grounded target. The cyclic voltammogram of $\mathbf{2}$ displayed a reversible redox wave at $0.79 \mathrm{~V}$ versus $\mathrm{Ag} / \mathrm{Ag}^{+}$, which was assigned to the oxidation of the nitroxide to the corresponding oxoammonium cation (Figure $4 \mathrm{~b}$ inset). The redox response did not show any significant deterioration even after 500 cycles. The anodic peak current was proportional to the sweep rate $v$, suggesting the contribution of redox profile for a surface-confined species and rapid electron transfer process of nitroxide radical (Figure $4 \mathrm{~b}$ ). The redox curves still exhibited a tailing shape, and the peak-to-peak separations $(\Delta E)$ also increased from 105 to $163 \mathrm{mV}$ with the sweep rate of 2 to $10 \mathrm{mV} \mathrm{s}^{-1}$, which suggested partial contribution from diffusion controlled redox response of the overlayed fibers, apart from the current collector. Previously, we have investigated the correlation of film thickness of 2 and its redox profile, and reported the transition (from surface-confined to diffusion-controlled) at a film thickness of $250 \mathrm{~nm} \cdot{ }^{10,26}$ Further optimization of the fiber diameters and configuration of overlayed fibrous membranes are still necessary; however, direct electrospinning of radical polymers without any radical defects is promising. Fibrous composite formation with current conductor additives such as multi-walled carbon nanotubes ${ }^{29}$

will also explore the potential of radical polymers as flexible and mechanically-durable battery electrodes.

\section{CONCLUSIONS}

By virtue of the inherent electroneutrality and high durability of nitroxide radical, the redox-active polymers were directly electrospun, for the first time, in one-step without additional processing aids such as polymer blends, post-doping, or protection/deprotection. The obtained micrometer-scale fibers had no defects on the radical moiety even under the application of high electric potential. Not only TEMPO-based polymers, but other redox-active, non-conducting polymers will be applicable for electro-spinning. In combination with a photocrosslinking reaction, electrospinning afforded insoluble, redox-active fibrous membranes, which are applicable for catalysts and electrode-active materials. This strategy is preliminary; however, further optimization of fiber formation (diameter), controlled deposition on the current collector and preparation of the composite with carbon nanotubes will lead to the new design of electrode-active materials toward the organic-based, flexible and wearable rechargeable batteries.

\section{ACKNOWLEDGEMENTS}

This work was partially supported by Grants-in-Aid for JSPS-NSF Research Collaboration Program and Scientific Research (19105003) from MEXT, Japan. This work was also supported by the US Army Research Laboratory and the US Army Research Office under Grant Number DAAD19-02-1-0275 Macromolecular Architecture for Performance (MAP) MURI.

1 Hawker, C. J., Bosman, A. W. \& Harth, E. New polymer synthesis by nitroxide mediated living radical polymerizations. Chem. Rev. 101, 3661-3688 (2001).

2 MacCorquodale, F., Crayton, J. A., Walton, J. C. \& Worsfold, D. J. Synthesis and electrochemical characterisation of poly(tempoacrylate). Tetrahedron Lett. $\mathbf{3 1}$, 771-774 (1990)

3 Sheldon, R. A., Arends, I.W.C.E., Ten Brink, G- J. \& Dijksman, A. Green, catalytic oxidations of alcohols. Acc. Chem. Res. 35, 774-781 (2002).

4 Nishide, H. \& Oyaizu, K. Toward flexible batteries. Science 319, 737-738 (2008).

5 Nishide, H. \& Suga, T. Organic radical battery. Electrochem. Soc. Interface 14, 32-38 (2005).

6 Nishide, H., Iwasa, S., Pu, Y.- J., Suga, T., Nakahara, K. \& Satoh, M. Organic radical battery: nitroxide polymers as a cathode-active material. Electrochim. Acta. 50, 827-831 (2004).

7 Nakahara, K., Iwasa, S., Satoh, M., Morioka, Y., Iriyama, J., Suguro, M. \& Hasegawa, E. Rechargeable batteries with organic radical cathode. Chem. Phys. Lett. 359, 351-354 (2002).

8 Suga, T., Sugita, S., Ohshiro, H., Oyaizu, K. \& Nishide, H. p- and n-Type bipolar redoxactive radical polymer: toward totally organic polymer-based rechargeable devices with variable configuration. Adv. Mater. 23, 751-754 (2011).

9 Suga, T., Ohshiro, H., Sugita, S., Oyaizu, K. \& Nishide, H. Emerging N-type redox-active radical polymer for a totally organic polymer-based rechargeable battery. Adv. Mater. 21, 1627-1631 (2009).

10 Oyaizu, K., Ando, Y., Konishi, H. \& Nishide, H. Nernstian adsorbate-like bulk layer of organic radical polymers for high-density charge storage purposes. J. Am. Chem. Soc. 130, 14459-14461 (2008).

11 Higuchi, M. Electrochromic functions of organic-metallic hybrid polymers. J. Nanosci. Nanotech. 9, 51-58 (2009).

12 Reneker, D. H. \& Chun, I. Nanometre diameter fibres of polymer, produced by electrospinning. Nanotechnology 7, 216-223 (1996).

13 Greiner, A. \& Wendorff, J. H. Electrospinning: a fascinating method for the preparation of ultrathin fibres. Angew. Chem. Int. Ed. 46, 5670-5703 (2007).

14 McKee, M. G., Layman, J. M., Cashion, M. P. \& Long, T. E. Phospholipid nonwoven electrospun membranes. Science 311, 353-355 (2006).

15 Hunley, M. T., Mckee, M. G. \& Long, T. E. Submicron functional fibrous scaffolds based on electrospun phospholipids. J. Mater. Chem. 17, 605-608 (2007).

16 Fukushima, S., Karube, Y. \& Kawakami, H. Preparation of ultrafine uniform electrospun polyimide nanofiber. Polym. J. 42, 514-518 (2010).

17 Nakashima, K., Tsuboi, K., Matsumoto, H., Ishige, R., Tokita, M., Watanabe, J. \& Tanioka, A. Control over internal structure of liquid crystal polymer nanofibers by electrospinning. Macromol. Rapid Commun. 31, 1641-1645 (2010). 
18 Kim, J.- K., Cheruvally, G., Choi, J.- W., Ahn, J.- H., Choi, D. S. \& Song, C. E. Rechargeable organic radical battery with electrospun, fibrous membrane-based polymer electrolyte. J. Electrochem. Soc. 154, A839-A843 (2007).

$19 \mathrm{Gu}$, Y., Chen, D. \& Jiao, X. Synthesis and electrochemical properties of nanostructured $\mathrm{LiCOO}_{2}$ fibers as cathode materials for lithium-ion batteries. J. Phys. Chem. B 109, 17901-17906 (2005)

20 Fan, Q. \& Whittingham, M. S. Electrospun manganese oxide nanofibers as anodes for lithium-ion batteries. Electrochem. Solid-State. Lett. 10, A48-A51 (2007).

21 Long, Y.- Z., Li, M.- M., Gu, C., Wan, M., Duvail, J.- L., Liu, Z. \& Fan, Z. Recent advances in synthesis, physical properties and applications of conducting polymer nanotubes and nanofibers. Prog. Polym. Sci. 36, 1415-1442 (2011).

22 Norris, I. D., Shaker, M. M., Ko, F. K. \& MacDiarmid, A. G. Electrostatic fabrication of ultrafine conducting fibers: polyaniline/polyethylene oxide blends. Synth. Met. 114, 109-114 (2000).

23 Li, D., Babel, A., Jenekhe, S. A. \& Xia, Y. Nanofibers of conjugated polymers prepared by electrospinning with a two-capillary spinneret. Adv. Mater. 16, 2062-2066 (2004).
24 Okuzaki, H., Takahashi, T., Miyajima, N., Suzuki, Y. \& Kuwabara, T. Spontaneous formation of poly(p-phenylenevinylene) nanofiber yarns through electrospinning of a precursor. Macromolecules 39, 4276-4278 (2006).

25 Yonekuta, Y., Susuki, K., Oyaizu, K., Honda, K. \& Nishide, H. Battery-inspired, nonvolatile, and rewritable memory architecture: a radical polymer-based organic device. J. Am. Chem. Soc. 129, 14128-14129 (2007).

26 Suga, T., Konishi, H. \& Nishide, H. Photocrosslinked nitroxide polymer cathodeactive materials for application in an organic-based paper battery. Chem. Commun. 1730-1732 (2007).

27 Colby, R. H., Fetters, L. J., Funk, W. G. \& Graessley, W. W. Constraint release in polymer melts: tube reorganization versus tube dilation. Macromolecules 24, 3873-3882 (1991).

28 McKee, M. G., Wilkes, G. L., Colby, R. H. \& Long, T. E. Correlations of solution rheology with electrospun fiber formation of linear and branched polyesters. Macromolecules 37, 1760-1767 (2004).

29 Hunley, M. T., Pötschke, P. \& Long, T. E. Melt dispersion and electrospinning of non-functionalized multiwalled carbon nanotubes in thermoplastic polyurethane. Macromol. Rapid Commun. 30, 2102-2106 (2009). 